\title{
Symptomatic Pelvic Kidney in Women at Childbearing Potential: Diagnostic Difficulties and Management in Urology Department of N'Djamena in Chad
}

\author{
Kimassoum Rimtebaye1, Edouard Hervé Moby Mpah², Arya Zarif Agah Tashkand1, \\ Franklin Danki Sillong'3 , Mignagnal Kaboro'1, Lamine Niang', Serigne Magueye Gueye ${ }^{4}$ \\ ${ }^{1}$ National General Referral Hospital of N'Djamena, N'Djamena, Chad \\ ${ }^{2}$ Laquintinie Hospital of Douala, Douala, Cameroon \\ ${ }^{3}$ Prostestant Hospital of N'Gaoundéré, N'Gaoundéré, Cameroon \\ ${ }^{4}$ General Hospital of Grand Yoff of Dakar, Dakar, Senegal \\ Email: melinarim@yahoo.fr
}

Received 23 November 2015; accepted 25 December 2015; published 28 December 2015

Copyright (C) 2015 by authors and Scientific Research Publishing Inc.

This work is licensed under the Creative Commons Attribution International License (CC BY).

http://creativecommons.org/licenses/by/4.0/

(c) () Open Access

\begin{abstract}
Introduction: We aim to report, from three observations, the diagnostic difficulties and complications, and ensure the management of pelvic kidney in women at childbearing age in the Urology Department of the National General Referral Hospital of N'Djamena. Pelvic kidney is due to an abnormality of the migration that can be associated or not with a malrotation. Its symptomatology is not specific, causing diagnostic difficulties and therapeutic errors. Observation: There were three observations of female patients aged respectively 26, 29 and 32 years who were taken care of for years in the gynecology department for pelvic inflammatory disease before being routed in urology. The diagnosis of pelvic kidney was retained on the basis of morphological examination (ultrasound, intravenous urography, CT urography). The patients have undergone nephrectomy; the postoperative aftermath was simple. Conclusion: Pelvic kidney is a rare disease. It is often discovered during autopsy. Symptoms are non-specific; nephrectomy is the treatment of choice in symptomatic cases.
\end{abstract}

\section{Keywords}

Pelvis, Iterative Cesarean, Malrotation, Nephrectomy, IVU

How to cite this paper: Rimtebaye, K., Mpah, E.H.M., Tashkand, A.Z.A., Sillong, F.D., Kaboro, M., Niang, L. and Gueye, S.M. (2015) Symptomatic Pelvic Kidney in Women at Childbearing Potential: Diagnostic Difficulties and Management in Urology Department of N'Djamena in Chad. Open Journal of Urology, 5, 246-253. http://dx.doi.org/10.4236/oju.2015.512039 


\section{Introduction}

Pelvic kidney is a malposition caused by lack of development constantly accompanied by a malrotation with one or more ectopic arteries and a ureter whose length depends on the kidney location [1]. A pelvic kidney is a kidney that had not migrated during renal ascent to the lumbar region. Its ureter is very short, thus promoting the vesico ureteral reflux and pyelonephritis. This is a rare urinary malformation, characterized by the presence of kidney in ectopic position below a plane passing through the iliac crests and fixed in this position by its vessels and the brevity of its ureter [2]. The pelvic kidney is most often discovered incidentally. It differs from renal ptosis. There are several types of kidney ectopias: simple ectopias taking place in the transverse direction (cross ectopia) and ectopias with symphyses. In practice the pelvic kidney poses various diagnostic problems. The symptomatology is not specific and causes diagnostic and therapeutic difficulties: infectious, lithiasic and obstetric complications. For women, clinical symptoms of a pelvic kidney are dominated by chronic pelvic pain simulating salpingitis, pelvic inflammatory disease, and appendicitis. The pelvic kidney may cause mechanical obstructed labor which can lead to iterative caesareans and tubal sterilizations. The treatment of choice for symptomatic pelvic kidney is nephrectomy [1] [3]-[5]. From three clinical observations, the authors aim to report the diagnostic difficulties and complications, and the management.

\section{Patients and Methods}

\subsection{Our Observations}

This was a retrospective descriptive study involving three female patients in reproductive age over a period of five years, from January 2008 to December 2013. The reasons for this study were explained to them and they consented as their medical records files and photographs will be anonymously used for science.

\subsubsection{Observation 1}

Madam X, 29, a mother of three living children, all cesarean delivery. At the third cesarean, she has undergone a tubal sterilization. The patient was followed for 15 years, in gynecological consultation for accute pelvic pain on a chronic background. These pains were treated unsuccessfully as a pelvic infection. Advised by her husband, health worker, she has consulted in urology for acute pelvic pain on a chronic background often associated with urinary burns.

The general condition was good. The vaginal examination combined with pelvic palpation has objectified a pelvic mass and a normal-sized uterus. The pelvic mass was located in the pouch of Douglas, behind the uterus and laterally to the left. The mobilization of the cervix did not involve that of the mass. The urinary culture had objectified an Escherichia coli infection. Blood glucose, blood urea nitrogen, creatinine were normal. The chlamydia serology was negative. Ultrasonography of pelvis and urinary tract had found: a normal sized uterus, homogeneous with a thin cavitary line; behind the uterus and laterally to the left, a mass with characteristics of a pelvic kidney (cortex, medulla and capsule were well separated) and the anatomical absence of the ipsilateral kidney in normal position (Figure 1).

IVU had objectified a left kidney in pelvic position with malrotation. Calyx are turned towards the center line opposite the promontory (Figure 2). The ureter, with short length, is obliquely downward and outward.

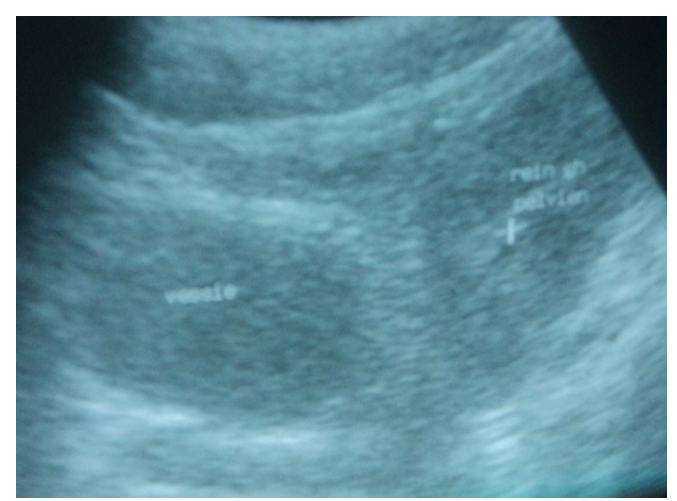

Figure 1. Ultrasound image of a pelvic left kidney. 
The epsilateral kidney is in normal position. Secretion and excretion of both kidneys were within normal delays.

The anti-infectious and analgesics treatments were administered without success. The patient has undergone a nephrectomy (Figure 3) for analgesics and anti-infectious aims. The operation was laborious due to the atypical vascularization of pelvic kidney.

Vascularization came from the external iliac vessels. A total nephrectomy carrying away the ureter until the vesicoureteral junction was performed. This kidney was normal size with a normal thickness parenchyma (Figure 4).

The postoperative aftermath was simple. The patient was regularly followed up over a period of two years without recurrence of pelvic pain or urinary burns before being declared cured.

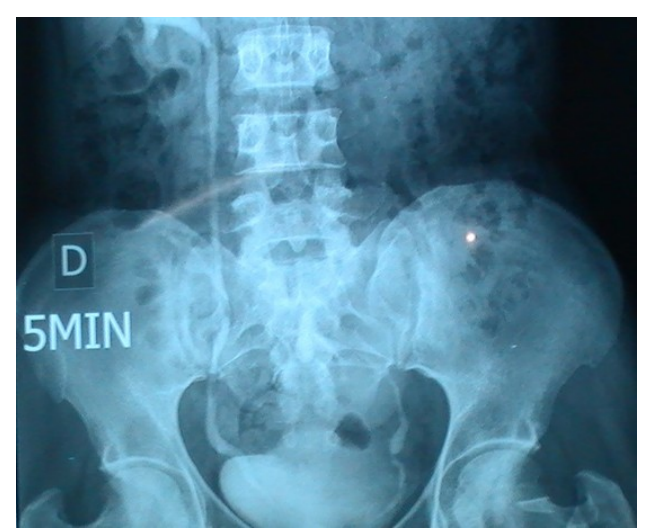

Figure 2. Left pelvic kidney with malrotation and short ureter at IVU.

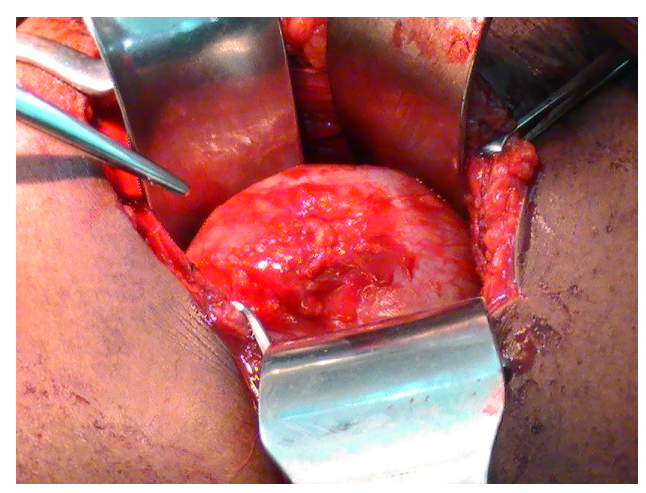

Figure 3. Per operative picture of a left pelvic kidney.

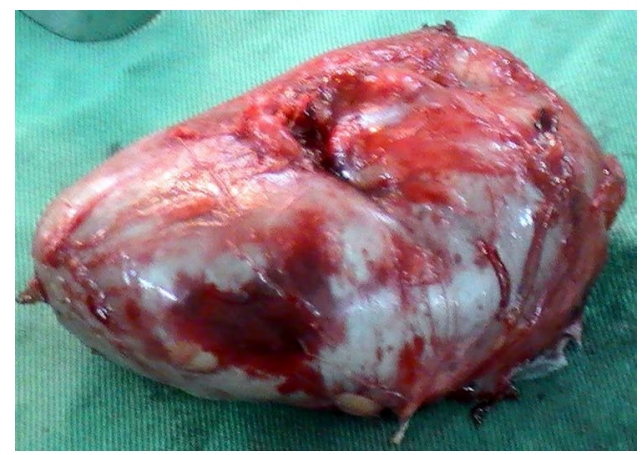

Figure 4. Operative specimen of a left pelvic kidney. 


\subsubsection{Observation 2}

Mrs XY, 32 years old, with a history of appendectomy 2 years before, has consulted in urology for a right inguinal pelvic pain. The pain was intense at times, often accompanied by fever, urinary burning and urinary urgency. Irradiation of this pain was either the external genitalia, sometimes to the hypogastrium, sometimes in hepatic region. The patient reported that these symptoms had caused an appendectomy without success. The patient had a good general condition. The vaginal examination combined with pelvic palpation has objectified a right pelvic mass of the size of an orange, painful and very mobile. Laboratory tests including blood count noted leukocytosis $9400 / \mathrm{ml}$, accelerated ESR, urinary culture had objectified a urinary tract infection with staphylococcus. Blood glucose, creatinine and blood urea nitrogen were normal. The morphological assessment including ultrasound, IVU, radiography of the urinary tract without preparation had gave the result below:

- Ultrasound: presence of a right pelvic mass whose echo structure evoked a right pelvic kidney, anatomical absence of the right kidney in right lumbar area, anatomical presence of the left kidney.

- Radiography of the urinary tract without preparation: presence of a calcic opacity at the promontory height (Figure 5).

- IVU: the plain abdominal X-ray confirmed the opacity objectified to the radiography of the urinary tract without preparation, cliches of secretion and excretion objectified in a right pelvic kidney. Calyxes were rounded, with convexity facing towards the promontory objectifying malrotation. The renal pelvis was the seat of obstructive lithiasis (Figure 6).

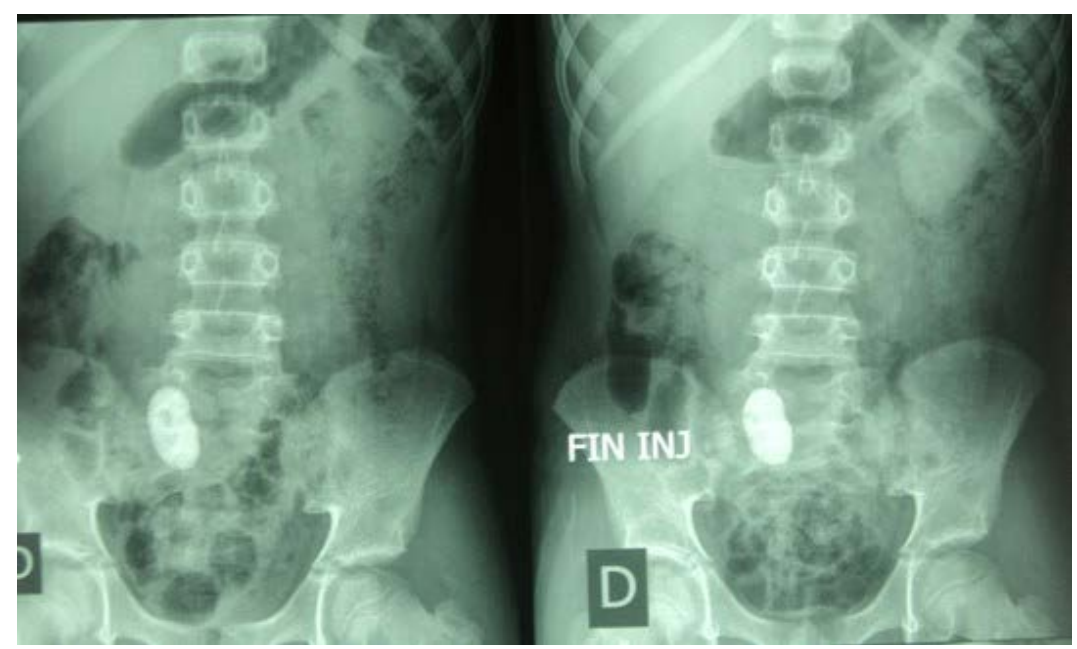

Figure 5. Nephrolithiasis in right pelvic kidney at the promontory height.

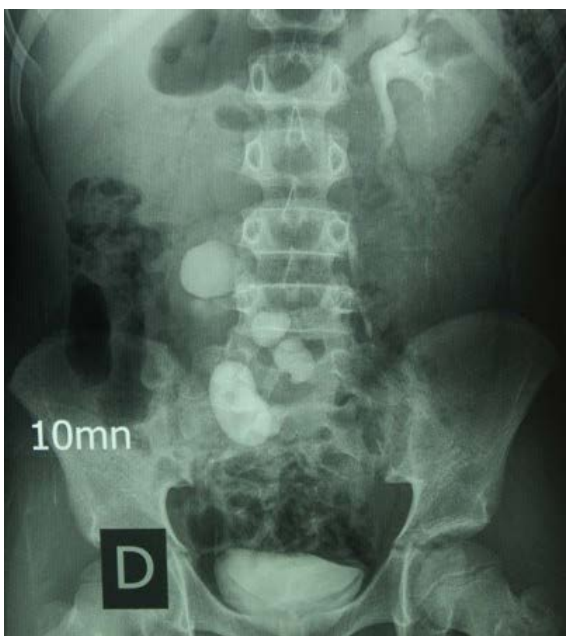

Figure 6. Caliceal dilatation upstream of the lithiasic obstacle on a pelvic kidney. 
The epsilateral kidney was morphologically and functionally good. The patient had received a total nephroureterectomy carrying off the lithiasis: it was about a lithiasic pyonephrosis (Figure 7). The vessels of the pelvic kidney were coming from the right external iliac vessels. The postoperative aftermath was simple. The exeat had been pronounced on the 7th day. The patient was taken care of regularly at the outpatient for a period of 18 months without complications or recurrence of pelvic pain before being declared cured.

\subsubsection{Observation 3}

Ms XX aged 26, mother of four living children, with a history of miscarriage, had been referred to a health facility, for management of a left ectopic kidney.

The disease onset would go back to June 2010, marked by the rupture of membranes during a six months pregnancy, followed by metrorrhagia causing a miscarriage. The next pregnancy causes pelvic pain motivating numerous consultations with the waning of which the diagnosis of an ovarian cyst associated with pregnancy had been selected. Several treatments were established without success. However, pregnancy was led forward to child birth. Given the persistence of pain despite the birth, the patient again consulted in gynecology. Ultrasound objectified a pelvic mass whose echostructure evoked a left pelvic ectopic kidney, hence the indication of a reference in urology. The general condition was good. On palpation, there was no lumbar contact, or renal sloshing; however painful sensitivity was noted in the pelvis.

Laboratory tests particularly creatinine, Urine culture, complete blood count, erythrocyte sedimentation rate, blood sugar and uric acid levels were normal.

A second ultrasound concluded that: Image pit left iliac in favor of an ectopic kidney. Anatomical absence of the left kidney in the normal position.

IVU objectified a left kidney in pelvic position without malrotation with shortness of the ureter (Figure 8). The epsilateral kidney was in normal anatomical position. Secretion and excretion of both kidneys were within normal delays.

CT urography: The cells wide shots had objectified two kidneys in different anatomical positions (Figure 9).

The right kidney is in normal anatomical position (lumbar sub costal), anatomically and functionally normal. The left kidney is in pelvic anatomical position, anatomically and functionally normal. The indication for an analgesic nephrectomy was retained and done. It was conducted a total nephrectomy taking off the ureter until the vesicoureteral junction. Vascularization came from the external iliac vessels. The kidney was normal size with a normal thickness parenchyma.

The patient was regularly monitored as outpatients for a period of 20 months without complications or recurrence of pelvic pain before being declared cured.

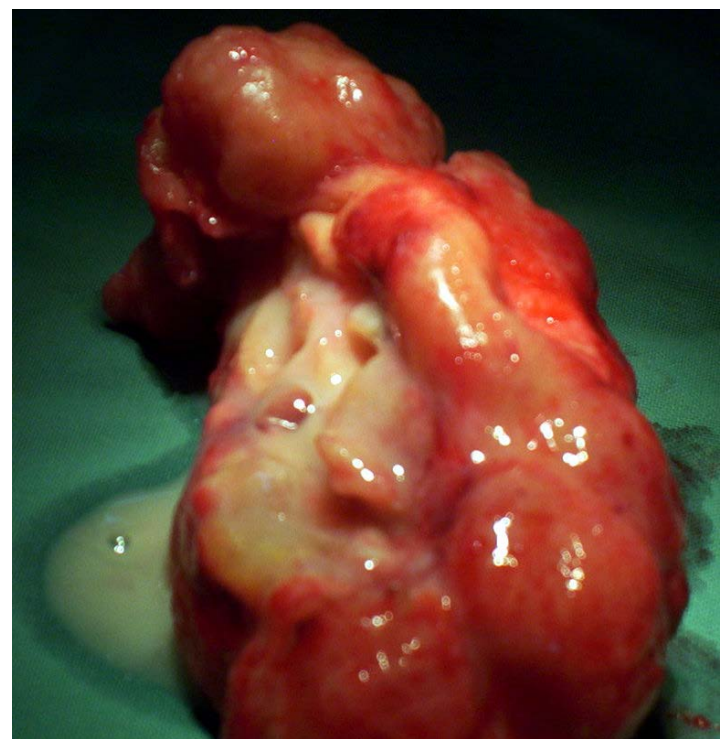

Figure 7. Lithiasic pyonephrosis. 


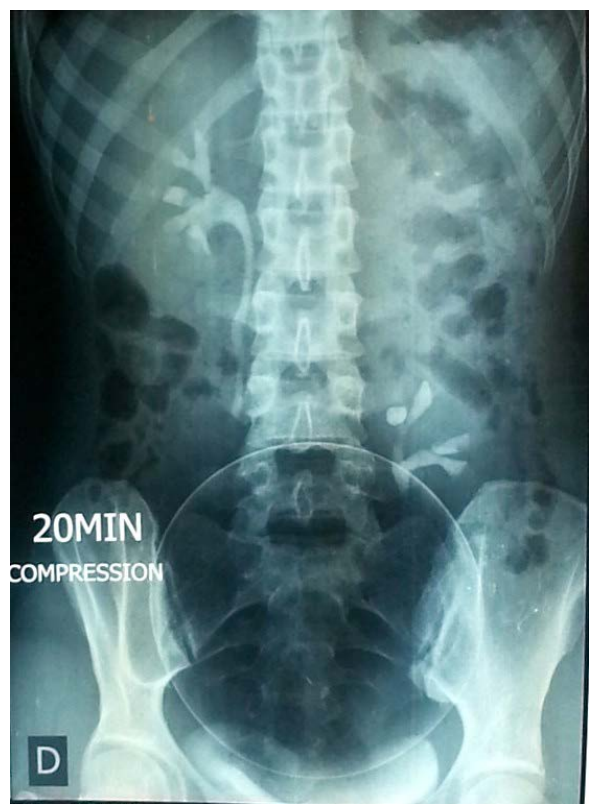

Figure 8. Left kidney without pelvic malrotation to IVU.

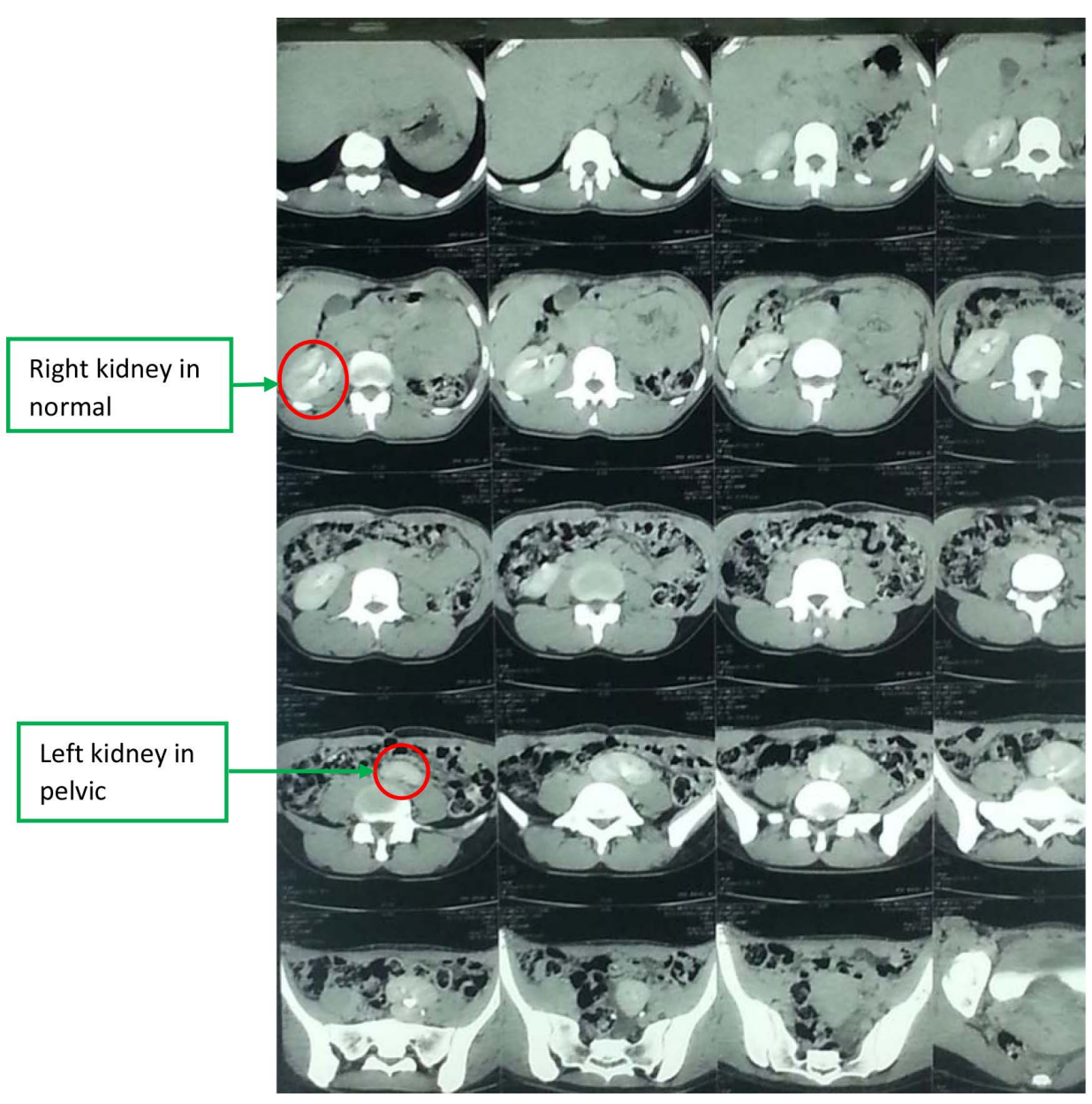

Figure 9. CT urography showing a pelvic left kidney. 


\section{Discussion}

The pelvic kidney is a very rare congenital anomalies of the kidney migration during embryogenesis. In 5 years, we have managed three cases. Proof of this scarcity is the lack of publications dealing with pelvic kidney management. The few published cases of pelvic kidney were discovered during a routine autopsy [1] [3]-[5]. Is this low frequency not it the witness of our ignorance of this disease with nonspecific symptoms and that its discovery can also be coincidental? The low specificity of symptoms is often a source of diagnostic and therapeutic errors detrimental to women at childbearing age. Clinical symptoms is most often that of a pelvic inflammatory disease or recurrent cystitis. This symptomatology is managed in gynecological practice. We think that the persistence of pelvic inflammatory disease symptoms despite a well conducted treatment should encourage to seek another etiological diagnosis, especially the pelvic kidney in women. It is rare if not exceptional to objectify a pelvic kidney through the vaginal examination combined with pelvic palpation. When a pelvic mass is objectified, it is most often suspected ovarian cysts, fibroids, ectopic pregnancy. In addition, pelvic pain are without any doubt the translation of renal colic on a pelvic kidney whose ureter shortness promotes vesicoureteral reflux and recurrent pyelonephritis. In our patients, pelvic pain is the main symptom. This pelvic pain, misdiagnosed as appendicitis, was originally an appendectomy without success. The patient of observation $n^{\circ} 1$ underwent three iterative caesarean section for obstructed labor with, third, the tubal sterilization. We believe this tubal sterilization was not justified. This obstructed labor is due to the presence of pelvic kidney which constitutes a mechanical obstacle. This lady could have sued her motherhood if the diagnosis of pelvic kidney was retained on time and a useful nephrectomy performed. Chronic pelvic pain, mechanical dystocia, recurrent pelvic infections in women at childbearing age should be a multidisciplinary management involving gynecologists, urologists and infectiologists.

Ultrasound performed in all three patients was used to guide the diagnosis. It gives the characteristics of a kidney namely: the regularity of its outlines, a good cortico medullary differentiation. It also gives a significant negative sign: the absence of anatomical ipsilateral kidney normal position. It confirms the presence of epsilateral kidney in normal anatomical position. It is desirable that the ultrasound is coupled with color Doppler ultrasound to better know the vascularization of the pelvic kidney which is often unpredictable. Indeed the pelvic kidney, like any ectopic kidney, takes its vascularization from arteries and veins of the vicinity unlike ptotic kidney.

Intravenous urography performed in our patients confirmed the diagnosis by demonstrating a functional pelvic kidney, with brevity of the ureter. IVU confirms the anatomical presence of the epsilateral kidney and its functionality which is very important information because of its functionality depends the heavy decision of nephrectomy.

The CT urography objectifies the kidney asymmetry position on serial sections which reinforce the diagnosis retained by ultrasound and IVU. These three morphological examinations (ultrasound, IVU, and CT urography) are essential for the definitive diagnosis of a pelvic kidney and its therapeutic indication. The magnetic resonance imaging is better suited to provide information on the vascularization of the pelvic kidney. Information of great interest for the surgical approach. For Zăhoi et al. angiography can better study the vascularization of an ectopic kidney [6].

The persistence and recurrence of pain alone justify nephrectomy that we call analgesic. The disappearance of these symptoms synonymous with relief and healing of patients reinforces the thesis of the indication of the nephrectomy. Multiple diagnostic errors could have been avoided by a holistic approach in the diagnostic process and surgical management. Pfannenstiel surgical technique which is going more obliquely upwards towards the iliac crest ipsilateral to the pelvic kidney has won our preference. An alternative would be the possibility to practice nephrectomy vaginally in the example of vaginal hysterectomy. A French study reported the possibility of laparoscopic nephrectomy with vaginal extraction would be somewhat less morbidly for obese patients [7] [8].

\section{Conclusion}

The pelvic kidney is an anomaly of kidney migration which can be associated or not to a malrotation. It is a rare disease often discovered at autopsy. The symptoms are not pathognomonic and not univocal. Diagnosis is based on imaging including: ultrasound, IVU, and CT urography. The total nephrectomy is the treatment of certainty when the clinical symptomatology is noisy. 


\section{References}

[1] Belsare, S.M., Chimmalgi, M., Vaidya, S.A. and Sant, S.M. (2002) Ectopic Kidney and Associated Anomalies: A Case Report. Journal of Anatomical Society of India, 51, 236-238.

[2] Zagyapan, R., Pelin, C. and Kürkçüoglu, A. (2009) A Retrospective Study on Multiple Renal Arteries in Turkish Population. Anatomy (International Journal of Experimental and Clinical Anatomy), 3, 35-39. http://dx.doi.org/10.2399/ana.09.007

[3] Somkuwar, S., Wahane, A. and Satpute, C. (2014) A Case Report of Failure of Ascent of Left Kidney with Non-Rotation. International Journal of Science and Research, 3, 1728-1730.

[4] Apoorva, D. and Lalitha, C. (2013) Unascended Left Kidney with Malrotation: A Rare Congenital Anomaly. International Journal of Science and Research, 2, 54-56.

[5] Singh, J., Singh, N., Kapoor, K. and Sharma, M. (2015) Bilateral Malrotation and a Congenital Pelvic Kidney with Varied Vasculature and Altered Hilar Anatomy. Case Reports in Medicine, 2015, Article ID: 848949. http://dx.doi.org/10.1155/2015/848949

[6] Zăhoi, D.E., Miclăuş, G., Alexa, A., Sztika, D., Pusztai, A.G. and Ureche, M.F. (2010) Case Report. Ectopic Kidney with Malrotation and Bilateral Multiple Arteries Diagnosed Using CT Angiography. Romanian Journal of Morphology and Embryology, 51, 589-592. www.ncbi.n/m.nih.gov/pubmed www.rjme.ro/RJME

[7] Paparela, P. and Golfierb, F. (2011) Laparoscopic Nephrectomy with Vaginal Extraction: A Real plus for Patients? (La néphrectomie laparoscopique avec extraction vaginale: Un vrai plus pour les patientes?) Progrès en UrologieFormation Médicale Continue, 21, F72-F76.

[8] Baldinia, A., Golfierb, F., Mouloude, K., Bruge Ansele, M.H., Navarroa, R., Ruffiona, A., et al. (2014) Day Case Laparoscopic Nephrectomy with Vaginal Extraction: Initial Experience. Urology, 84, 1525-1528. http://dx.doi.org/10.1016/j.urology.2014.06.084 